\title{
An android app for receiving, storing and exchanging blood pressure data way bluetooth, OCR and gesture input
}

\begin{abstract}
Telemetry home, mobile technologies, and software application, has a positive effect onthe monitoring and management of some medical control parameters, and help to be more effective in the treatments, measuring blood pressure at home is becoming increasingly use by both clinicians and patients. This work implements a way to obtain information directly from commercial blood pressure devices, using application programming interface, without to have used the proprietary APP of the brand, use of the optical character recognition and the use of gestures using the fingers to write each of the blood pressure values; systole, diastole, pressure, date and time and send these data to a Web-DataBase way SMS message
\end{abstract}

Keywords: blood pressure, OCR, gestures, APP, treatment, optical character recognition, hypertension
Volume 6 Issue 2 - 2020

\author{
José de Jesús Sandoval-Palomares, Javier \\ Yañez-Mendiola \\ Department of Applied research, CIATEC, México
}

\begin{abstract}
Correspondence: José de Jesús Sandoval-Palomares, Department of Applied research, CIATEC, A.C, Omega 20I, Industrial Delta 37545, León Gto, México, Tel 47771000I,
\end{abstract} Email jsano@ciatec.mx

Received: November 08, 2019 | Published: June 08, 2020
Abbreviations: BP, blood pressure; SMS, short message messages; API, application programming interface; OCR, optical character recognition; APP, application; SFF, structure frame format

\section{Introduction}

Monitoring the high blood pressure (BP) help to prevent causes of stroke and other cardiovascular complications. ${ }^{1,2}$ some national and international guidelines also recommend home monitoring in certain circumstances, in this way, telemetry home and mobile devices technologies has a positive effect in the management of some medical control parameters and help to be more effective in the treatment, measuring BP at home is becoming increasingly use by both clinicians and patients. ${ }^{3-9}$ Studies apply monitoring BP, watched patients felt more aware of their heart failure condition, less anxiety, and more empowered, the clinicians were able to manage their patients heart failure conditions more effectively, because they had physiological data reported to them frequently to help in their treatment. ${ }^{10,11}$ Whereas some commercial systems for BP have significant research and an Application (APP) is available, other systems lack apps, ${ }^{12,13}$ then exist the opportunity to improve hypertension control, through easier logging of repeated BP measurements, better connectivity with healthcare providers and reminder alerts for medication recommendations. ${ }^{14}$ Mobile health apps are an ideal tool for monitoring and tracking longterm health conditions. Apps need to succeed in ensuring the privacy, security, and safety of the health data entrusted to them. ${ }^{15}$

We consider that nowadays all the portable BP measurement device have a monochromatic display to report the measurement performed, classically: systole, diastole, pressure, date and time; OMRON, A\&D MEDICAL, ALMEDIC, IHEALTH, IPROVEN, to mention brands. Some of these brands provide improve devices giving the communication feature by wireless WIFI or Bluetooth technology as the most widespread, for communicated to smartphones or mobile device with IOS or Android operating system and a proprietary APP of the brand, where users can enter the data obtained and carry out continuous monitoring. The last trend in this aspect is the use of smart watches, which contain an optical sensor that allows obtaining various medical parameters, including BP, not necessarily very accurate, as in their indications they express them themselves. We found a method to implement a mechanism, to obtain information directly from commercial BP without having to use the proprietary APP of the brand, like the use of the Optical Character Recognition (OCR) to obtain each of the BP values; systole, diastole and pressure and completing with the use of gestures when using the fingers to write the value and help for people with some visual impairments. With these methods to obtaining BP values from different devices and have the possibility to send these data to a Web-DataBase way SMS message, is the objective of this project, which also includes implement a universal repository and not depend on brands to monitor and control the levels of BP, covering an opportunity where users and clinicians do not have to be changing platforms, APP or repository to help with this problem.

\section{Methods}

The APP interface was developed for Spanish speakers using all the screen to help the quick and direct access to all the options menu. The menu contain the next options; General Data ( that get the general information of user ), Manual ( allow the manual number capture of systole, diastole, pulsation, date and hour ), Camera/OCR ( get the image from the screen of a commercial medical BP device ), Bluetooth ( allow get the information from a commercial BP monitor), Data consulting ( shows in a chart the pressure information or shows in a table the pressure information), SMS (send the actual records to an external SMS receptor), Delete Results (which removes current data from the database ), Website (which refer to the http address where can view the information in the Web-Database), and Exit (close the APP). For the OCR recognition, we used the internal camera from the android device, the Tesseract SDK for Android, in the OCR we only convert the numbers obtained from the images, and only recognize 1 to 3 continuous digits. For manual entry we implement an interface, where the user can use a pencil o directly his finger, follows a digit Ds-font as a stroke guide. The external connection with the bluetooth was with a commercial BP monitor M7-Intelli-IT from Omron Brand. The APP was coded for Android 6.0 operative system, over Android 
studio 3.4 and the internal database in SQLite, the Figure 1, shows the general process in the APP.

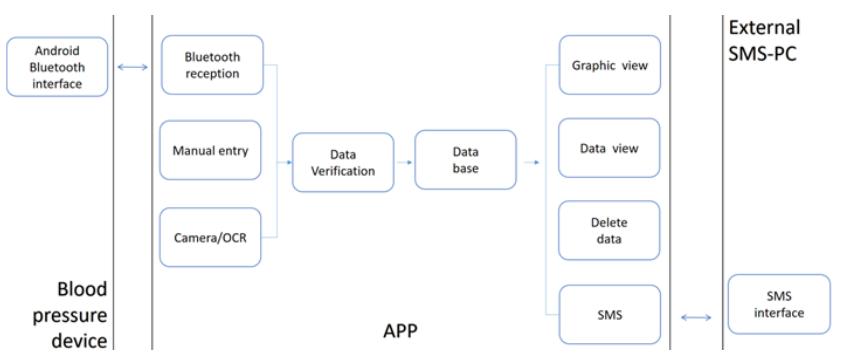

Figure I General process of the android APP for exchanging blood pressure data.

\section{Direct manual entry}

For manual entry we impended a screen, where the user can write each of number for systole, diastole and pulse using the classic scroll up-down, in this way, the user select each digit in each scroll Figure 2. We comment than all the values have a maximum and minimums range, initially is configured like systole 100 y $140 \mathrm{mmHg}$ (millimeters of mercury), diastole $60 \mathrm{y} 90 \mathrm{mmHg}$ and 60 to $100 \mathrm{ppm}$ (pulse per minute), this values is checked in OCR, finger-gesture writing and direct manual entry, if data input are outs of this ranges, shows an error message input data and ask for new data.

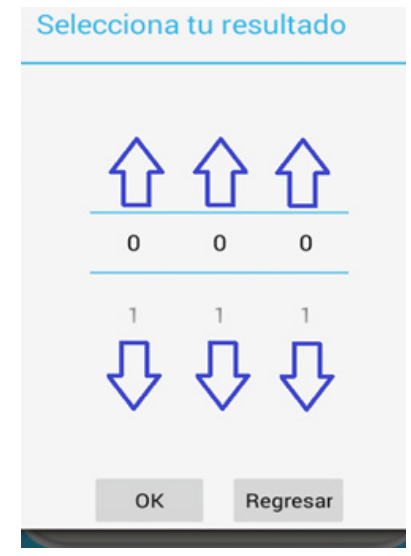

Figure 2 Direct manual entry screen.

\section{Entry by finger-gesture writing}

For the implement of a finger-based number input method on touchscreen, for normal people with some visual impairments. We implement a digit by digit input with the finger; a user sketches a gesture that passes through the lines in the corresponding segment code for digit 0 to 9 , as a stroke guide. for this purpose, an interface with two sections was developed, in the upper section it was divided into three areas to identify the position of the digits, the lower section shows how the digit type number should be drawn Ds-font as the visual stroke guide Figure 3. In the upper section, the first area to the left corresponding to the hundreds, the next to the right the tens and the next to the right and last for the units, this allowed simplifying both the user and technically the entry of a numerical value using finger-gesture. The algorithm for capture works as follows, in the screen, specifically in the gesture capture area, the user is asked to draw the digit following the tracing guide corresponding to number
0-9 and the value of hundreds, tens and units independently, if the user makes a mistake or wants to delete his stroke, they could double click on the image of the digit and it will be deleted and will allow a new capture. The previous procedure has the consideration that if the user enters a digit in the hundreds or tens and the other missing sections do not enter anything, the value is considered to be zero and by extension any missing digit will be zero, for example, entering hundreds the digit, "2_ ", will be interpreted as 200, "1_5", as 105 , in some cases it would be similar, "_ 8 " would be 80 and for only units " 9" is interpreted as only 9, this helps to write fewer digits to introduce. The drawn lines corresponding to each digit is saved in an image that is converted to gray and sent to the Tesseract API that interprets it and returns the identified digit and is displayed to the user for confirmation or correctness. When finished, the user presses the verification button and the value will be displayed in a message, the user verifies it and selects the "Ok" or "return" button, if the option is "return", the user can modify the value by following the procedure again described, with the option is "OK" the interpreted digits are stored in the SQLite database.

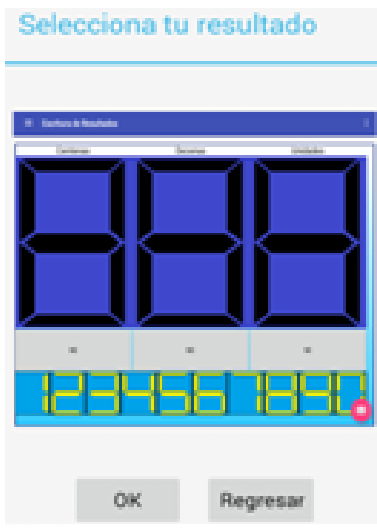

Figure 3 Entry by finger-gesture writing screen.

\section{Optical character recognition (OCR)}

To apply OCR, we perform text detection and text recognition using Tesseract API, who is a well-known open source OCR library that can be integrated with Android apps. One important activity was the Tesseract installation and configuration of the API of Tesstwo, this API and instructions was obtained from https:/github.com/ rmtheis/tess-two site. We have used the English version of tessdata, in this case because we used only the number recognition, was configured and processed the jpg image to an Android bitmap with $500 \mathrm{~kb}$ maximum and the set of the text using getUTF8 function. In the Figure 4 shows graphically the process performed for OCR recognition, which is described below. The user activates the APP option to capture values by OCR, then the camera service is activated, for this operation, we use the standard Android camera function, the user places the camera of the mobile device in a way that the image of the commercial BP monitor and its numbers are seen, Figure 4A. In the screen, Figure 4B, of the mobile device two sections are shows, the section one with the full image, with a small rectangle with red edges, which helps to place the number, at down the section two , is shown the region of the rectangle as a zoom type for a better visualization (the correct placement of the area where the number is observed is $100 \%$ controlled by the user), the user captures the image Figure $4 \mathrm{C}$, this is converted to grayscale, this conversion is limited only the red rectangle area of the image where the number is observed, this 
image is processed with the API of Tesseract Figure 4D to obtain the numeric value, the identified value is shows and in a message and Figure $4 \mathrm{e}$, if the numerical data is correct, the user touches the screen and requests the following numbers, a total 3 numbers are requested, corresponding to the systole, diastole and pulse, finished the capture , the data is stored in the SQLite database, Figure 4F, and the screen with the obtained numbers is presented in the APP screen, Figure $4 \mathrm{G}$.

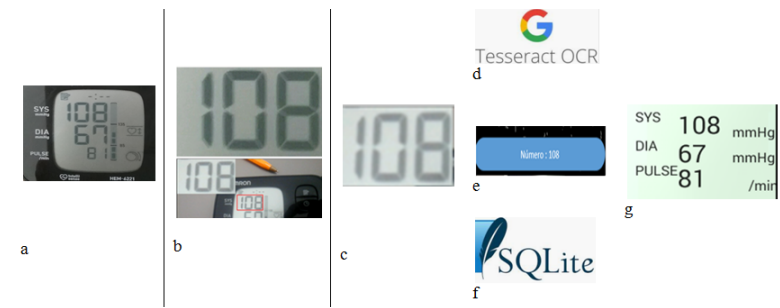

Figure 4 graphically process performed for OCR recognition, capturing by image the digits of a portable blood pressure measurement device.

\section{Testing process}

\section{Final prototype}

The final APP menu is shows in the Figure 5, the interactive with the big size menu with different icons and colors to allow users select easily the option. The direct manual entry capture option is shows in the Figure 6, in Figure 6a ask the user the systole, diastole and pulse data, and Figure $6 \mathrm{~b}$ shows data input scroll mechanism, the user does click in number and used the arrows to select the required number, Figure $6 \mathrm{c}$ shows the input data number. In the entry by finger-gesture writing, the user can have written 3 digits and one guide digits was implemented to follow the correct way and the recognition be faster, the Figure 7A-7D shows these test steps.

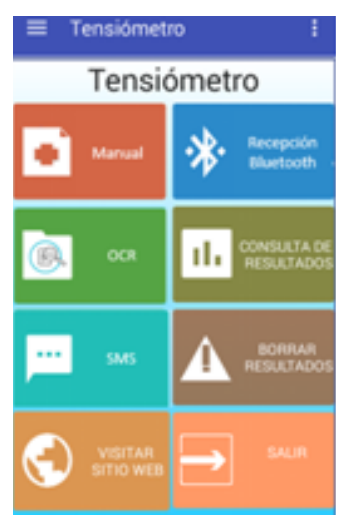

Figure 5 General menu of the android APP for exchanging blood pressure data.

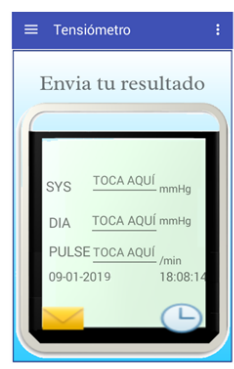

a
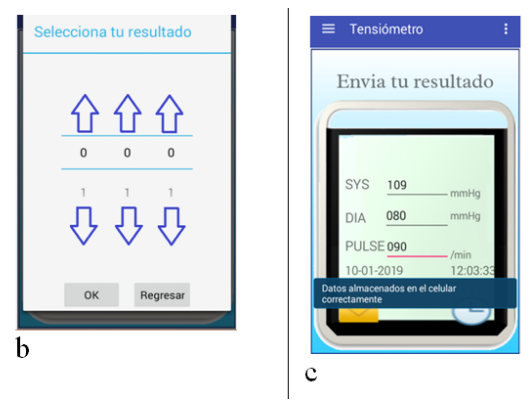

QW

Figure 6 Interface of APP for manual input of blood pressure data.
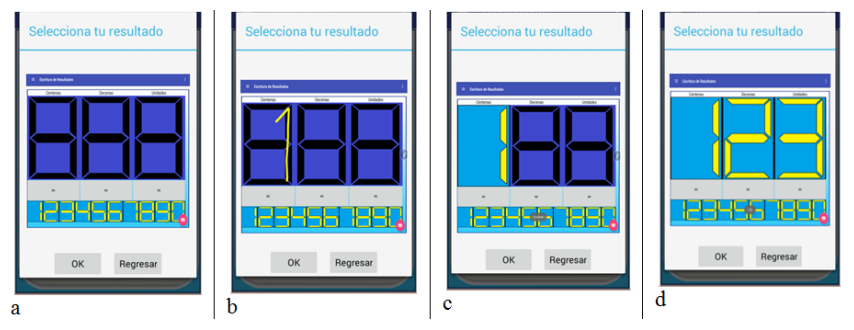

Figure $\mathbf{7}$ Interface of APP for gesture input by fingers of blood pressure data.
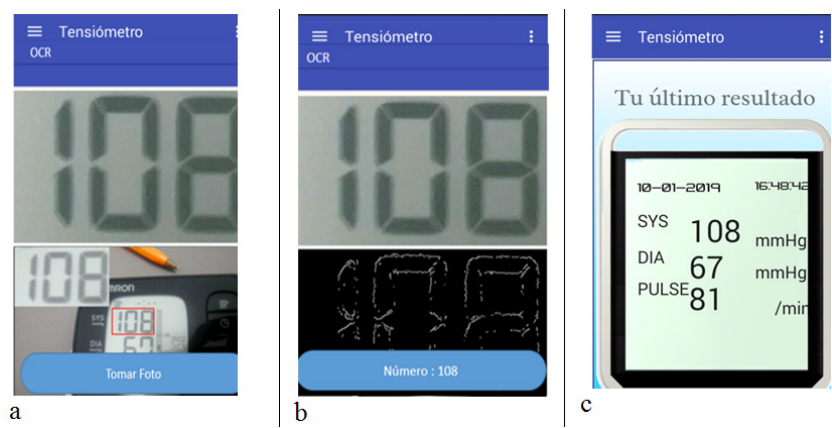

Figure 8 Interface of APP for OCR input by fingers of blood pressure data

In the optical character recognition interface implementation was in 3 steps, in test we obtain the image with the number Figure 8A, a red rectangle to boundary the number and other area to view the zoom of this number, a second step to make the recognition of number by OCR technic, finally shown the number in the screen, Figure 8B. Both manual and OCR option each number recognized was send to a screen like the Figure 8C, and wait for the user confirmation.

To verify the correct functionality of the system, different pilot tests were run, a mobile device android 6.0 was turned on and off and the APP was connected and disconnected and it was tested different functionalities to verify correct functionality, we followed user case testing from TI methodology, which allowed verifying that the APP complied with each of the functionalities designed and encoded. Verifying the capture by gesture writing was tested several times the numbers input and some possible variations to verify the implement recognition algorithm and translate it variations correctly. In the OCR test, we used different positions of the Android device camera and BP screen and found the possible variations when the image will be captured to help in a best algorithm recognizer of OCR technique. The SMS was verified when taking the data from the database and correctly formed the data frame and it was received by the external SMS, in this case a confirmation of reception of the external web was not implemented so as not to complicate this process. The tests were performed by 3 users who are currently in control of their blood pressure, helping to identify recommendations on their use and functionality.

\section{Results}

In the direct manual entry, did not present a major problem when entering the digits, the 3 users intuitively entered these and understood the mechanics without further explanation. The support of guides that was implemented in the method of gestures with the finger or pencil help a lot to resolve some problems, like recognizing the different ways in which a number is written, for example the number " 1 " which in some cases came to recognize it as " 7 ", or the " 5 " since it was written more as a "S" and was not recognized and the variations of " 4 " also presented problems, the digit Ds-font guide helped these problems 
were drastically solved. In the case of OCR recognition, helped recognize only the digits from 0 to 9 , which reduces the processing time, however, there were more difficulties in taking the image since this should have been centered on a rectangular region as an area to recognize by OCR, besides, that helped avoid glare or light refractions in this area. To obtain the digits by OCR, some problematic nontechnical conditions were found, such as the quality of recognition may vary depending on the light conditions of the image, sharpness, camera resolution, type and font size of BP commercial, associated with the position and brightness when the photo is taken, to achieve the highest possible quality, it was important to center the text and focus the image correctly. Test users commented that they did not find it difficult to understand the operation of the system and the APP is intuitive, useful and simple to use.

\section{Conclusion and future works}

This work presents the proposal to interconnect elements that are traditionally disconnected; we integrated these multiple elements to a single platform such as BP monitoring devices, APP, Database, patient users and medical specialists. So on we implement useful characteristics, by incorporating communication to BP monitoring devices into a non-proprietary APP and obtaining their data, the use of this direct data capture or through finger gestures, which allows older o some visual impairments users not to have to change their habits when writing a number and can transmit it to medicals, capture by OCR is another novelty, which expands the possibilities of obtaining information on BP measurement devices that do not have communication, helping to reduce the cost and be more universal when obtaining this information. By last other important novelty is the sending of blood pressure data by SMS to an external WebDatabase, and data could even be sent from remote places without an internet connection, connection that is indispensable in the other proprietary and non-proprietary APPs. We need to improve the way you take the image for the OCR, since it was only tested with the gray screen that most devices have, however others are emerging with color screens and higher quality. Another element to review is the problem with the brightness when taking the photos, one idea is to work with the brightness and contrast, and test how this could improve OCR recognition. In addition, a new version of the APP is considering adding voice recognition for data entry. This project opens the panorama, as it does not depend solely on the applications that commercial systems can offer, in addition to the fact that the type of BP device used doesn't matter, clinicians and users may have only one interface to control and monitoring blood pressure levels.

\section{Funding details}

Consejo Nacional de Ciencia y Tecnología, México (National Council for Science and Technology, CONACYT) and TecnoGadget, S. A de C.V. Mexican company.

\section{Acknowledgments}

None.

\section{Conflicts of interest}

Authors declare that there is no conflict of interest.

\section{References}

1. Riis J, Nordestgaard BG, Jensen GB, et al. Secular trends in risk of stroke according to body mass index and blood pressure. 1976-2017. Neurology. 2019;93(14):e1397-e1407.

2. Sarah Lewington, Robert Clarke, Nawab Qizilbash, et al. Prospective Studies Collaboration. Age-specific relevance of usual blood pressure to vascular mortality: a meta-analysis of individual data for one million adults in 61 pro-spective studies. Lancet. 2002;360:1903-1913.

3. Chobanian AV, Bakris GL, Black HR, et al. The seventh report of the joint national committee on prevention, detection, evaluation, and treatment of high blood pressure. JAMA. 2003;289:2560-2571.

4. Blood pressure control by home monitoring: meta-analysis of randomised trials. 2004.

5. De Vries AE, van der Wal $\mathrm{MH}$, Nieuwenhuis $\mathrm{MM}$, et al. Health professionals' expectations versus experiences of internet-based telemonitoring: survey among heart failure clinics. $J$ Med Internet Res. 2013;15(1):e4.

6. Parati, G, Stergiou G, Asmar R. et al. European Society of Hypertension Practice Guidelines for home blood pressure monitoring. J Hum Hypertens. 2010:24:779-785.

7. Stergiou G, Mengden T, Padfield PL, et al. Working Group on Blood Pressure Monitoring of the European Society of Hypertension, Self monitoring of blood pressure at home. Br Med J. 2004;329:870-871

8. Teemu JN, Marjo-Riitta H, Jouni J, et al. Home-Measured Blood Pressure Is a Stronger Predictor of Cardiovascular Risk Than Office Blood Pressure. Hypertension. 2010;55:1346-1351.

9. Martínez-Pérez B, de la Torre-Díez I, López-Coronado M. Mobile health applications for the most prevalent conditions by the world health organization; review and analysis. J Med Internet Res. 2013;15(6):e120.

10. Seto E, Leonard KJ, Cafazzo JA, et al. Mobile phone-based telemonitoring for heart failure management: a randomized controlled trial. $\mathrm{J}$ Med Internet Res. 2012;14(1):e31.

11. Seto E, Leonard KJ, Cafazzo JA, et al. Perceptions and experiences of heart failure patients and clinicians on the use of mobile phone-based telemonitoring. J Med Internet Res.2012;14(1):e25.

12. Martínez-Pérez1 B, De la Torre-Díez I, López-Coronado M, et al. Mobile Apps in Cardiology: Review. JMIR Mhealth Uhealth. 2013;1(2).

13. Reddy R, Majmudar M, Dhopeshwarkar N, et al. Mobile health apps preferences and practice among ambulatory cardiovascular patients. Future Cardiology. 2018;14(5):381-388.

14. Goldberg EM, Levy PD. New Approaches to Evaluating and Monitoring Blood Pressure. Curr Hypertens Rep. 2016;18:49.

15. Knorr K, Aspinall D, Wolters M. On the Privacy, Security and Safety of Blood Pressure and Diabetes Apps. IFIP Advances in Information and Communication Technology.2015;455. 